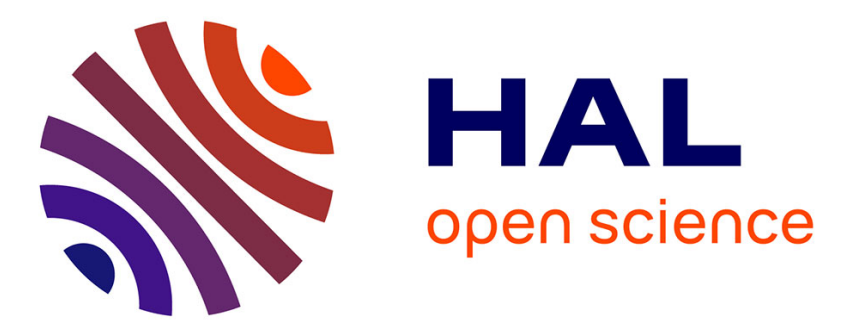

\title{
Comparer deux espaces urbains du commerce transnational (Le Caire et Oran), ou comment gérer les écarts entre théorie de la comparaison et pratique sur le terrain
}

Anne Bouhali

\section{To cite this version:}

Anne Bouhali. Comparer deux espaces urbains du commerce transnational (Le Caire et Oran), ou comment gérer les écarts entre théorie de la comparaison et pratique sur le terrain. e-migrinter, 2015, Mobilités, circulation migratoire et transnationalisme au prisme des réflexions méthodologiques de jeunes chercheur(e)s en sciences sociales, 13, 10.4000/e-migrinter.556 . halshs-01739646

\author{
HAL Id: halshs-01739646 \\ https://shs.hal.science/halshs-01739646
}

Submitted on 21 Mar 2018

HAL is a multi-disciplinary open access archive for the deposit and dissemination of scientific research documents, whether they are published or not. The documents may come from teaching and research institutions in France or abroad, or from public or private research centers.
L'archive ouverte pluridisciplinaire HAL, est destinée au dépôt et à la diffusion de documents scientifiques de niveau recherche, publiés ou non, émanant des établissements d'enseignement et de recherche français ou étrangers, des laboratoires publics ou privés. 
(e- migrinter

\section{e-Migrinter}

$13 \mid 2015$

Mobilités, circulation migratoire et transnationalisme au prisme des réflexions méthodologiques de jeunes chercheur(e)s en sciences sociales

Comparer deux espaces urbains du commerce transnational (Le Caire et Oran), ou comment gérer les écarts entre théorie de la comparaison et pratique sur le terrain

Anne Bouhali

revues.org

Éditeur

UMR 7301 - Migrinter

Édition électronique

URL : http://e-migrinter.revues.org/556

DOI : 10.4000/e-migrinter.556

ISSN : 1961-9685

\section{Référence électronique}

Anne Bouhali, «Comparer deux espaces urbains du commerce transnational (Le Caire et Oran), ou comment gérer les écarts entre théorie de la comparaison et pratique sur le terrain », e-Migrinter [En ligne], 13 | 2015, mis en ligne le, consulté le 30 septembre 2016. URL : http://e-

migrinter.revues.org/556; DOI : 10.4000/e-migrinter.556

Ce document a été généré automatiquement le 30 septembre 2016.

Tous droits réservés 


\title{
Comparer deux espaces urbains $d u$ commerce transnational (Le Caire et Oran), ou comment gérer les écarts entre théorie de la comparaison et pratique sur le terrain
}

\author{
Anne Bouhali
}

\section{Introduction}

1 La démarche comparative fait aujourd'hui pleinement partie des sciences sociales, et notamment de la géographie urbaine, que ce soit au sein de travaux collectifs ou dans des travaux plus solitaires comme la thèse. Néanmoins, la plupart des chercheurs ayant mis en œuvre cette démarche entreprennent toujours de justifier leur choix méthodologique, en particulier lorsqu'il s'agit d'une thèse. Car faire le choix de la comparaison pose plusieurs questions. Tout d'abord, pourquoi proposer un travail comparatif, et donc multiplier les terrains, plutôt que de se concentrer sur une étude de cas qui pourrait être également tout à fait pertinente? Comment construire en géographie un objet de recherche selon cette approche comparative? Enfin, quels sont les apports de la comparaison, à la fois dans la façon de mener plusieurs terrains de front et dans les résultats qu'elle produit, et quelles en sont les limites ? Cette contribution propose ainsi de répondre modestement à ces questions, en partant d'une démarche réflexive assumée, résultat de travaux toujours en cours.

2 Notre recherche doctorale porte sur deux marchés anciens et populaires situés au cœur de deux grandes villes du monde arabe, Oran et Le Caire, et qui sont toutes deux alimentées en produits de consommation courante ce que certains auteurs appellent les «nouvelles routes de la soie» (Simpfendorfer, 2009), autrement dit les réseaux 
commerciaux de la «mondialisation par le bas » (Peraldi, 2001, 2002 ; Tarrius, 2002). Cette forme de mondialisation, qualifiée également de "mondialisation non hégémonique " (Mathews, Alba Vega, 2012), est un phénomène qui touche les Suds, mais également les Nords, et à la tête duquel on trouve désormais de véritables professionnels qui proposent aux consommateurs des classes populaires et des petites classes moyennes des produits de consommation courante à bas prix, et réalisés en Asie du Sud-Est et orientale (babioles, vêtements, ustensiles de cuisine, etc.). Notre thèse cherche à analyser les impacts des flux de produits "made in Asia» très divers et des réseaux commerçants sur des espaces urbains «discrets» (Pliez, 2007) car situés à l'écart des flux financiers majeurs de la mondialisation.

3 Ce choix méthodologique a ainsi soulevé de nombreuses questions une fois sur le terrain, et lors des allers-retours entre nos deux terrains. Il s'agira ainsi, après avoir exposé dans un premier temps les motifs et les contraintes qui nous ont fait choisir la comparaison comme méthodologie de recherche, d'expliquer dans un deuxième temps la construction de cette comparaison. Dans un dernier temps enfin, nous montrerons quels sont les apports de la comparaison à notre travail, mais également les difficultés et les limites rencontrées.

\section{Pourquoi comparer deux dispositifs du commerce transnational dans le monde arabe?}

Le choix de la comparaison dans le cadre de notre thèse de géographie ne s'est pas imposé au début de notre recherche. Il est en effet le résultat de contraintes externes. Néanmoins, de choix par défaut, le comparatisme s'est avéré un outil méthodologique pertinent pour mener nos terrains et pour construire notre cadre de recherche.

\section{De la monographie à la comparaison, un choix sous contrainte}

5 Au départ, faire un travail de comparaison entre plusieurs terrains ne faisait pas partie de notre méthodologie de recherche, bien que plusieurs thèses de géographie urbaine soutenues ces dernières années en avaient montré toute la pertinence (Spire, 2011; Fleury, 2007 ; Choplin, 2006 ; Morelle, 2004 ; Salin, 2002). Lors de la construction de notre projet de thèse, il s'agissait uniquement de travailler sur une étude de cas, Le Caire, et ainsi de proposer une monographie des espaces urbains du commerce transnational dans la capitale égyptienne, articulés au dispositif commercial d'échelle nationale et transnationale. Le Caire étant la première capitale d'Afrique $d u$ Nord en termes économiques et démographiques, réaliser une monographie paraissait un choix tout à fait pertinent et qui nécessitait un travail de terrain approfondi tout à fait réalisable le temps d'une thèse. La capitale égyptienne avait de plus déjà fait l'objet de travaux préliminaires sur la question de la "mondialisation discrète " (Pliez, 2007) et sur son inscription non seulement dans les marchés populaires du centre historique cairote, mais aussi dans les espaces portuaires, portes d'entrée des marchandises "made in China» (Doron, 2006). Nous bénéficions ainsi de premiers éléments de recherche sur les espaces urbains du commerce transnational en Égypte.

Cependant, la révolution de 2011 qui a mis fin en quelques jours au règne du président Moubarak a également causé l'annulation de notre premier terrain de recherche 
exploratoire d'avant-thèse. Le risque était alors grand de devoir travailler pendant les trois prochaines années sur un terrain épisodiquement voire complètement fermé à la recherche. La solution d'aller explorer d'autres terrains et de construire un travail de comparaison s'est alors dessinée. Les travaux de Philippe Gervais-Lambony avaient ouvert la voie à la comparaison en géographie urbaine à la fin des années 1990, suite à la publication de son étude comparée de la citadinité à Harare et à Lomé (Gervais-Lambony, 1994). Plusieurs thèses comparatives avaient également été soutenues depuis le début des années 2000 et montraient ainsi qu'il était envisageable, théoriquement, mais aussi concrètement, de multiplier les terrains au cours des quelques années dédiées à la recherche doctorale.

7 Progressivement, l'idée s'est donc imposée d'étudier non pas le dispositif commercial cairote lui-même, mais plutôt de se pencher sur l'étude générale de dispositifs commerciaux transnationaux ancrés sur les routes de la «mondialisation discrète " à l'intérieur du monde arabe. Il s'agissait d'étudier plutôt le lien entre commerce transnational et fabrique de la ville, et notamment la transformation de centralités commerciales en ville, plutôt que d'étudier une ville particulière face au commerce transnational, en vue de monter davantage en généralité sur les questions de commerce transnational et de fabrique urbaine.

\section{Croiser les terrains, mais quels terrains?}

8 Ne voulant pas abandonner le terrain cairote malgré la conjoncture politique sensible, nous souhaitions trouver un autre terrain permettant d'étudier l'inscription dans la «mondialisation discrète » de places marchandes intra-urbaines et ses conséquences sur le tissu urbain originel. L'Algérie est apparue alors comme un choix de terrain tout à fait pertinent.

9 Cette décision s'explique dans un premier temps par le contexte de construction de la recherche française en sciences sociales portant sur la «mondialisation par le bas ». En effet, le cas algérien a été étudié par les travaux pionniers de sociologues, d'abord ceux d'Alain Tarrius (2000) puis ceux de Michel Peraldi $(2001 ; 2002)$ qui ont tous deux travaillé sur le « comptoir marseillais» (Tarrius, 1995) et ses activités commerciales informelles. Les deux chercheurs ont montré que les activités économiques de cette "centralité immigrée » étaient tributaires de la mise en place d'un commerce « au cabas », dit aussi "commerce à la valise", entre l'Algérie et la France. Ce commerce, mené par les «fourmis» de la mondialisation travaillant et vivant entre la France et l'Algérie, bénéficiait de l'état de pénurie qui régnait alors dans l'Algérie socialiste des années 1980 . Ces migrants transnationaux circulaient entre les deux rives de la Méditerranée et répondaient ainsi aux besoins de consommation de la population algérienne en lui procurant des produits achetés en France. Suite à la fermeture des frontières françaises aux Algériens à la fin des années $1980^{1}$, les travaux de recherche dirigés par Michel Peraldi $(2001 ; 2002 ; 2005)$ ont montré que ces petits commerçants se sont ensuite tournés vers d'autres espaces marchands plus favorables à cette forme de commerce « au cabas", allant toujours plus à l'est de la Méditerranée et achetant désormais des conteneurs entiers en Turquie et à Dubaï, avant de partir en Asie du Sud-Est, considérée comme «la » source des produits de consommation courante vendus à bas prix dans le monde entier. Le commerce transnational en Algérie avait ainsi fait l'objet de plusieurs publications avant et pendant ma thèse (Spiga, 2002 ; Belguidoum, Pliez, 2012 ; Kerdoud, 
2012), ce qui me faisait bénéficier d'un socle théorique et empirique solide, utile pour ensuite me saisir du cas égyptien, moins étudié.

Dans un second temps, l'Égypte et l'Algérie sont les deux pays les plus peuplés d'Afrique du Nord et sont, de fait, les deux principaux centres de consommation d'articles « made in China ». De plus, ce sont deux «États entrepôts » (Igué, Soulé, 1993) qui ont développé et développent aujourd'hui encore, un commerce de réexportation de ces produits importés de Chine en direction d'autres pays de la région nord-africaine, notamment vers la Tunisie et la Libye dans le cas algérien (Belguidoum, Pliez, 2012; enquêtes de terrain de juillet 2012), et vers l'Afrique sub-saharienne dans le cas égyptien (Bava, Pliez, 2009).Dans un dernier temps, travailler en Algérie me permettait de bénéficier d'un accueil de la part d'universitaires et de centres de recherche algériens, ce qui a facilité mon entrée sur le terrain.

11 Néanmoins, si étudier la question du commerce transnational dans ces deux pays se justifiait pleinement, les marchandises « made in China » étant très visibles tant en milieu urbain que rural depuis la fin des années 1990 et le début des années 2000, les deux villes choisies ne se ressemblent guère. En effet, Oran et Le Caire diffèrent évidemment et ostensiblement par leur taille et leurs fonctions. Le Caire, capitale politique, économique et culturelle écrasante domine tout le pays et concentre au sein de son agglomération, le Grand Caire, entre 16 et 17 millions d'habitants. Oran, quant à elle, est la deuxième agglomération algérienne et comprend dix fois moins d'habitants (1,5 million d'habitants), elle ne joue aucun rôle politique - le pays étant extrêmement centralisé au bénéfice de la capitale algéroise - et voit son rayonnement économique essentiellement limité aux régions de l'ouest du pays.

12 Un critère évident permettant la comparaison serait peut-être l'appartenance à la même aire culturelle, le monde arabe. Mais est-ce suffisant, et surtout, nécessaire pour mener à bien une comparaison? Elodie Salin et Marie Morelle soulignent en effet que l'appartenance à une même aire culturelle n'est pas un critère nécessaire pour comparer, et ne justifie pas en soi le choix de la comparaison (Salin, 2002 ; Morelle, 2004). Marcel Detienne ajoute que "comparer au plus loin» est souvent encore plus frappant et davantage producteur de réflexions que «comparer au plus proche» (Detienne, 2002). Pour pouvoir comparer, il faut construire des catégories qui rendent possible la comparaison, que l'on compare « au plus proche » comme c'est notre cas, ou « au plus loin ».

\section{De la comparaison à la construction de comparables}

13 Il s'agit dans cette deuxième partie d'expliquer ce que signifie " comparer ", et comment comparer. Pour cela, nous nous sommes beaucoup appuyés sur les travaux de Marcel Detienne $(2000,2002)$, mais aussi sur plusieurs thèses en géographie urbaine qui ont choisi une démarche comparative, et qui ont ainsi largement nourri notre réflexion théorique.

\section{Construire des comparables}

Qu'est-ce que la comparaison en sciences sociales? Si l'on en croit Marcel Detienne, il s'agit moins de comparer, autrement dit, de faire un travail d'analogie qui viserait à faire 
émerger des ressemblances et des divergences, que de construire des comparables qui vont permettre ensuite de monter en généralité et de s'interroger sur une catégorie, ellemême divisible en « une série de composants conceptuels de plus en plus subtils, lesquels renvoient à autant de questions sur le terme initial » (Detienne, 2002, 74). Dans notre cas, la comparaison entre Le Caire et Oran devient pertinente lorsque l'on s'intéresse, non pas aux villes en elles-mêmes ou à leur agglomération, mais aux dispositifs commerciaux transnationaux que sont les places marchandes irriguées par des circulations de produits « made in China », et dont, d'ailleurs, Oran et Le Caire n'ont pas l'exclusivité.

Comment mettre en œuvre une telle comparaison en tant que géographe? Nous proposons de nous intéresser au dispositif commercial comme catégorie qu'on peut décomposer en objets géographiques propices à la comparaison. Un dispositif commercial transnational est, dans nos études de cas, une place marchande, elle-même centralité intra-urbaine plus ou moins ancienne (dès le $\mathrm{X}^{\mathrm{e}}$ siècle pour Le Caire, le début $\mathrm{du} \mathrm{XX}^{\mathrm{e}}$ siècle pour Oran), aujourd'hui alimentée par des circulations transnationales de produits qui la transforment, non seulement en remodelant son tissu urbain, mais aussi en modifiant les modes de consommation de la clientèle et en faisant intervenir une série d'acteurs, depuis le consommateur appartenant à une classe plutôt populaire en passant par le vendeur de rue, le commerçant au détail, en demi-gros ou en gros, l'importateur et le gestionnaire urbain. Enfin, ce dispositif commercial doit se lire à plusieurs échelles : celle intra-urbaine, celle de la ville et de l'agglomération, celle du réseau commercial de places marchandes à l'échelle régionale, nationale, et enfin transnationale.

En définitive, il ne s'agit pas tant de comparer deux villes a priori incomparables que de comparer un même phénomène qui s'inscrit dans plusieurs villes; en considérant l'objet d'étude, à savoir la place marchande transformée par le commerce transnational, la comparaison reprend tout son sens. C'est donc le thème conducteur de la recherche qui permet la comparaison et la mise en perspective de ces deux villes, dans notre cas celui de la mise en place d'un dispositif commercial transnational ancré sur les routes de la mondialisation discrète. Une fois ces éléments identifiés, il est possible de mettre en miroir les deux contextes urbains et comparer les places marchandes d'Oran et du Caire à travers des sous-catégories.

\section{Comparer une forme urbaine du commerce transnational : le cas du centre commercial}

17 Un exemple de construction d'un comparable serait celui du centre commercial ${ }^{2}$, une forme urbaine de consommation qui s'est massivement développée au cœur des deux places marchandes de notre étude depuis le début des années 2000, alors qu'elle était jusqu'alors présente uniquement dans les espaces de consommation à destination des catégories sociales supérieures, en Égypte en particulier (Abaza, 2006). Comparer la présence de ce nouveau type commercial et son inscription dans le tissu urbain est intéressant en tant qu'il s'agit là d'un marqueur majeur de la mondialisation des modes de consommation qui accompagne la diffusion des produits « made in China " en direction des petites classes moyennes et populaires égyptiennes et algériennes. Le comparable « centre commercial » peut alors lui-même être divisé en plusieurs éléments comparatifs.

Tout d'abord, les noms donnés aux centres commerciaux et aux galeries commerçantes sont significatifs d'histoires commerciales différentes entre l'Algérie et l'Égypte d'un point de vue temporel et géographique. Côté algérien, les enseignes font souvent 
référence à des routes anciennes de la «mondialisation discrète" et à des places marchandes qui ont été les premières dans lesquelles se sont rendus les commerçants algériens suite à la fermeture des frontières françaises. Ainsi, un centre commercial qui compte parmi les premiers érigés à Medina J'dida à Oran a pour nom " Istamboul ${ }^{3}$ ", rappelant ainsi que la ville turque est une des places marchandes dans lesquelles les commerçants algériens se fournissent depuis les années 1990 et jusqu'à aujourd'hui des produits textiles (linge de maison, vêtements) de bonne qualité (Pérouse, 2007 ; enquêtes 2012, 2013 et 2014). On pourra également relever le fait que les premiers marchés de produits d'importation ont été appelés des « Dubaï ", en référence au rôle de hub joué par l'Émirat. Côté égyptien, les enseignes font référence à d'autres places marchandes plus anciennes, antérieures à Istanbul et à Dubaï. Nous avons en effet noté plusieurs noms de centres commerciaux faisant directement référence à la Syrie ou à Gaza, deux territoires anciennement pourvoyeurs de vêtements et de tissus. Mais c'est actuellement la référence aux modèles du Golfe qui prédomine, avec des noms comme le mall «Le Royaume " dont l'enseigne montre une femme portant un hidjab à la mode du Golfe faisant directement référence à cette région. Cette référence aux pays du Golfe en général témoigne de la forte influence de la religiosité de la région sur la société égyptienne, et notamment sur les classes populaires. Cela rappelle également que ces vêtements pudiques pour femme étaient achetés à Dubaï, et le sont encore parfois, l'Émirat étant un avant-poste des productions chinoises en direction du monde arabe et musulman. Certains commerçants interrogés prétendent encore que ces articles, les abaya noires, qui sont les robes noires couvrant entièrement le corps de la femme mais qui laissent le visage découvert, sont produites en Arabie Saoudite ou à Dubaï bien qu'elles viennent d'Asie, comme une part importante des marchandises vendues sur le marché. Cette référence aux Émirats a gagné Medina J'dida il y a peu. Un centre commercial a en effet ouvert en 2013 avec pour nom «centre commercial Les Émirats arabes unis», ce qui indique selon nous une diffusion de ce modèle culturel en direction des consommatrices et des consommateurs algériens.

Un autre objet de comparaison intéressant concerne les noms employés pour désigner ces nouveaux magasins. Au Caire, deux termes coexistent : à côté du terme générique " markaz tujari », c'est-à-dire littéralement "centre commercial», on trouve très fréquemment les termes «mall » et "center», deux termes anglais écrits en caractères latins, mais aussi et surtout transcrits en caractères arabes. À Oran, on trouve de même l'expression « markaz tujari » aux côtés du terme «bazar », ou beaucoup plus rarement aux côtés du terme "galerie ». Ces dénominations sont particulièrement signifiantes. En ce qui concerne le cas égyptien, cela montre la percolation des modèles de consommation internationaux, plutôt occidentaux, mais revisités via les pays du Golfe (Abaza, 2006), des classes supérieures vers les classes populaires, avec l'utilisation de termes anglais qui sont passés dans la langue arabe du quotidien. Côté algérien, les trois termes ne sont pas parfaitement synonymes. "Galerie " n'a été trouvée qu'une seule fois dans tout le périmètre du marché et désigne un seul grand magasin de Medina J'dida, qui semble ancien, et qui ferait peut-être référence au modèle français de la galerie commerçante. L'expression « centre commercial » désigne un seul et même magasin, neuf, vendant des produits d'importation tandis que "bazar» désigne uniquement les immeubles commerciaux abritant en leur sein des boutiques indépendantes, qui bien souvent vendent le même produit (Kerdoud, 2012). Il faut noter que "bazar » dans le contexte égyptien signifie en principe une galerie marchande rassemblant de nombreux vendeurs d'articles, mais que, de nos jours, le terme a largement perdu cette signification et 
s'emploie souvent pour désigner une boutique d'antiquaire ou un brocanteur ; le terme " mall » est devenu dominant et est complètement passé dans le langage courant, ce qui serait peut-être encore un signe de la diffusion des pratiques de consommation égyptiennes des classes supérieures vers les classes populaires, ce que confirme un entretien réalisé avec un commerçant dans le district de la quincaillerie dans le Caire historique ${ }^{4}$. Cette personne, grossiste et importateur en quincaillerie, membre d'une grande famille de commerçants, confirme cette idée de percolation des modèles de consommation des classes supérieures vers les quartiers populaires. En 2013, cet homme était à la tête d'un vaste chantier de construction d'une tour commerciale de douze étages en plein cœur historique, destinée à être un «mall» spécialisé dans les articles de décoration et de bricolage pour la maison. Quand nous lui avions demandé pourquoi il avait choisi de faire construire une tour tout en verre bleuté, il nous avait répondu que les quartiers chics de la ville, Madinat Nasr, Nasr Gedida/Heliopolis, et Mohandessin - qui concentrent une large part des «malls» à destination des classes supérieures, avaient tous des tours de commerce, et qu'aujourd'hui il fallait proposer la même chose dans le vieux Caire. Construire de grands immeubles commerciaux est donc un signe évident de modernité, à l'intérieur de quartiers populaires. En construisant des sous-catégories, des " composants de plus en plus subtils» pour reprendre l'expression de Marcel Detienne $(2002,74)$, il nous est alors possible de comparer Oran et Le Caire, en tout cas en ce qui concerne les objets géographiques.

Ainsi, en construisant des comparables, comme nous le proposons brièvement ici, il nous est possible de mettre en évidence certains invariants liés au fonctionnement de places marchandes transnationales irriguées par des flux d'articles «made in China». Pour reprendre une expression de Marie Morelle, il s'agit par la comparaison de «mettre à jour d'éventuels mécanismes et au-delà l'universalité possible du phénomène » (Morelle, 2004, 17) et dans notre cas, de commencer à «dresser le portrait » de l'ancrage urbain de la mondialisation discrète lorsqu'elle s'inscrit dans deux centralités urbaines et commerciales anciennes, toutes deux situées dans des villes qui se ressemblent pourtant peu ou pas.

\section{Apports et difficultés de la démarche}

En menant ce travail de comparaison, nous essayons de « mieux comprendre une ville par l'étude d'une autre, et inversement » (Gervais-Lambony, 1994, 12), et de mieux nous saisir de notre objet d'étude, le commerce transnational, en faisant des allers-retours entre Oran et Le Caire, afin de relever les invariants qui seraient propres aux places marchandes populaires et discrètes mondialisées en Afrique du Nord. Aller d'un terrain à l'autre est ainsi extrêmement fructueux ; cela suscite à la fois étonnement et prise de distance lorsque l'on change de terrain, et cela permet parfois de mieux comprendre un aspect d'une ville après l'avoir observé et étudié dans une autre. Cependant, comme nous le verrons, cela ne se fait pas sans certains écueils.

\section{Allers-retours entre Oran et Le Caire : étonnement et prise de distance d'un terrain à l'autre}

L'apport le plus sensible des va-et-vient entre nos deux terrains, et dont témoignent également de nombreuses thèses de géographie, aura été tout d'abord l'étonnement que 
produit un retour sur le terrain après avoir séjourné, même brièvement, sur le second. Philippe Gervais-Lambony écrit ainsi que, "dans le cadre d'une comparaison, l'observation d'autres lieux facilite un regard différent sur le lieu étudié " (GervaisLambony, 2003, 23). Naviguer entre deux terrains a souvent été révélateur d'objets, de thèmes de recherche, de questions que nous ne nous étions pas immédiatement posés lors d'un premier terrain.

Par exemple, la question de la présence de vendeurs ambulants dans les espaces publics, autour du marché ou bien à l'intérieur du marché dans les rues commerçantes, ne nous avait pas paru saillante lors de notre premier séjour au Caire à la fin de l'hiver 2011/2012. $\mathrm{Au}$ premier abord, le marché situé à la sortie de la station de métro Ataba nous avait semblé être un marché officiel et régulier, et non une occupation illégale de l'espace public par de jeunes commerçants, comme c'est en réalité le cas. L'organisation de ce marché en effet semblait obéir à un ordre, à l'image de marchés organisés par les pouvoirs publics: chaque table est aménagée avec des stores en bois ou en tissus permettant de moins souffrir du soleil et de la chaleur qui se font sentir dès la fin du printemps, chaque commerçant conserve son emplacement d'un jour à l'autre et laisse même ses marchandises sur sa table pendant la nuit, ficelées sous des couches de carton. Mais c'est grâce au terrain suivant, effectué en Algérie à l'été 2012, que la question de l'occupation des espaces publics, illégale notamment, par les petits commerçants s'est posée. En effet, en Algérie, le commerce à la sauvette est une réalité extrêmement importante, que ce soit dans la rue devant les boutiques ou sous la forme d'un commerce ambulant pratiqué par des "vendeurs informels", comme on les appelle au quotidien. Il joue le rôle de palliatif à la crise économique, car c'est une solution venue « du bas » pour répondre au sous-emploi qui touche de nombreux jeunes ${ }^{5}$. Cette réalité sociale est tellement prégnante dans la ville algérienne que ces jeunes sont appelés « hittistes », de " hit » qui signifie en arabe « mur », et désigne par extension ceux qui « tiennent le mur », autrement dit, les jeunes désœuvrés. Nous avons pu ainsi comprendre les relations entre ces jeunes vendeurs ambulants et les commerçants installés dans les boutiques de Medina J'dida, les premiers se fournissant parfois en produits invendus des saisons précédentes et vendus moins chers auprès des seconds, voire même les premiers payant une petite redevance aux seconds en vue d'avoir l'autorisation tacite d'installer leurs charrettes, tables ou cartons devant la vitrine des commerçants installés. Cette question de « l'ambulantage » et de l'occupation de la rue a été ainsi un nouvel axe de recherche lors de notre retour au Caire en 2012, ce qui nous a permis de progressivement complexifier notre regard sur le marché cairote qui, étant extrêmement vaste comparé à Medina J'dida en termes de surface, de nombre de commerçants, de consommateurs, de boutiques et de vendeurs de rue, a tendance à diluer parfois nos observations et à ne pas se laisser saisir au premier coup d'œil. En ce sens, nous ne pouvons qu'être d'accord avec Philippe Gervais-Lambony lorsqu'il écrit que «l'apport de la comparaison est alors d'éclairer un objet par rapport à l'autre. En un lieu, certaines questions se posent à un moment donné. On ne pense à les poser à propos de l'autre lieu que parce que l'on a fait ce va-et-vient... Cela, bien sûr, en évitant l'écueil qui consisterait à assimiler deux objets : il s'agit bien de garder à l'esprit qu'ils sont différents » (Gervais-Lambony, 2003, 24).

De même, ces allers-retours entre Oran et Le Caire nous ont permis de nous recentrer progressivement sur notre sujet de recherche : la question urbaine. Le point de départ de nos deux premiers terrains à Oran en novembre 2011, puis au Caire en février-avril 2012 était la question urbaine. Mais nous avons progressivement suivi une autre piste, celle de 
l'approvisionnement des places marchandes étudiées, et donc des réseaux de places marchandes du commerce transnational vers la Chine. L'objectif était ainsi de saisir l'organisation de ce commerce à l'échelle nationale et métropolitaine. Le deuxième terrain en Algérie, qui nous a permis d'aller d'Oran jusque dans les grandes villes commerçantes de l'est algérien, vers une des principales sources d'importation des articles « made in China » dans le pays, s'inscrivait dans cette même idée de comprendre la centralité régionale replacée dans le contexte plus large du commerce transnational en Algérie. Cependant, ce déplacement de places en places sur les routes algériennes du commerce transnational nous a mené à envisager à nouveau la question de la fabrique même de ces places marchandes. En effet, se déplacer de marchés en marchés nous a permis de constater que la construction même du tissu urbain - les magasins, les centres commerciaux - était considérablement affectée par ce commerce. Le troisième terrain algérien au printemps 2013 a été l'occasion de vérifier le dynamisme urbain de ces territoires du commerce: le nombre d'immeubles neufs à l'intérieur du périmètre de Medina J'dida à Oran avait visiblement augmenté en quelques mois. Lors de notre second et dernier terrain en Égypte en 2013, puis de notre quatrième et dernier terrain algérien en 2014, il s'agissait donc de revenir aux lieux, aux marchés et à leur tissu bâti, à la fabrique de la ville par le commerce, après avoir longtemps essayé de comprendre le fonctionnement de ces marchés comme places de concentration de flux puis de redistribution vers d'autres espaces, ruraux et urbains.

\section{La comparaison comme voie détournée : lire une ville pour éclairer l'autre}

25 Un autre apport important de la pratique de la comparaison est son utilité pour éclairer des aspects parfois difficiles, voire obscurs, d'un terrain grâce à un détour par un autre terrain. Il ne s'agit plus ici de renouveler son regard, de redécouvrir certains aspects d'un terrain après avoir séjourné sur l'autre, mais plutôt de chercher des réponses à des difficultés rencontrées sur le premier terrain.

Par exemple, dans le cadre de notre recherche, la question de la circulation et de l'achat de devises en vue de pratiquer l'importation internationale est un enjeu quotidien pour le commerce transnational en Égypte comme en Algérie. Un des moyens d'avoir accès à des sommes souvent conséquentes, si ce n'est colossales à en croire certains grands commerçants enquêtés en Égypte et en Algérie, est de se fournir sur le marché noir, car le marché officiel n'est pas en mesure de répondre à ces besoins, notamment parce qu'une partie de ces activités d'import est illégale. Des recherches ont été publiées sur les deux contextes, l'Égypte ayant eu un marché noir du change très développé dans les années 1990 (Moisseron, Youssef, 2004), et l'Algérie présentant une véritable économie au noir parallèle à l'État (Benbouzid, 1999; Bellal, 2008). Autant les commerçants égyptiens étaient peu diserts à ce sujet, autant les commerçants algériens en parlaient librement, le marché noir de la devise étant une réalité très présente et surtout visible dans les villes algériennes, cette activité se pratiquant parfois dans l'espace public au vu et au su de tous ${ }^{6}$. Nous-mêmes, en tant que consommateurs, avons fait l'expérience à notre niveau du change au noir dans toutes les villes algériennes où nous avons eu l'occasion de séjourner. Le dialogue était ainsi plus facile et plus direct avec les commerçants algériens qui, une fois la confiance établie, étaient relativement prêts à nous expliquer leurs pratiques et le fonctionnement du marché noir de l'euro, ainsi que leurs « trucs » pour pouvoir faire du 
commerce avec l'étranger de façon discrète et fructueuse. Les informations obtenues en Algérie nous ont ensuite permis de mieux formuler nos questions en revenant au Caire, de faire des remarques plus pointues montrant une certaine connaissance des pratiques. Les commerçants égyptiens nous répondaient alors plus volontiers. Comprendre les échanges financiers du côté algérien nous a ainsi permis d'avoir plus d'informations côté égyptien.

\section{Les risques de la comparaison}

Cependant, aux côtés de ces atouts indéniables de la comparaison pour la pratique du terrain, nombreuses et nombreux sont les géographes qui, après avoir mené un travail comparatif, mettent en garde contre les écueils, voire les risques, de la comparaison. Nous avons également eu l'occasion de rencontrer ces difficultés à maintes reprises sur nos terrains.

Tout d'abord, nous avons pris conscience de la difficulté à appliquer concrètement, une fois sur le terrain, une méthodologie strictement symétrique sur chacun des terrains. Même si le cadre méthodologique était le même - mêmes grilles, quoique "grossières ", pour les entretiens semi-directifs, même travail d'observation et d'immersion à différents moments pour mieux saisir les rythmes urbains, entre autres - nous n'étions pas toujours en mesure de faire exactement des choses semblables au Caire ou à Oran. Il a fallu adapter les grilles d'entretien, qui étaient de fait plutôt des lignes directrices que des grilles très figées. Les acteurs interrogés n'étaient pas toujours les mêmes selon la difficulté à les approcher et à établir un dialogue de confiance. En bref, il a fallu s'adapter et adapter notre méthodologie qualitative aux aspérités du terrain.

L'autre difficulté à l'œuvre dans le travail comparatif, et peut-être une des difficultés essentielles, consiste en la maîtrise inégale des deux terrains. Choisir de comparer deux terrains, c'est dans une certaine mesure multiplier les éléments bibliographiques, chercher à maîtriser deux contextes économiques, politiques et sociaux différents qui permettent pourtant de mieux saisir et maîtriser les particularités de chaque espace urbain étudié. C'est également développer des réseaux de connaissances dans deux espaces différents. Le chercheur finalement se retrouve face à deux terrains bien évidemment complexes, qui mériteraient chacun, peut-être, une étude en particulier. Certains chercheurs ont ce parti pris assumé de revendiquer l'un des deux (ou des trois) terrains comme étant le terrain principal, «son » terrain, pour reprendre l'expression d'Armelle Choplin (2006). C'est le cas d'Antoine Fleury, pour qui le point de départ de sa recherche a été Paris, un " ancrage », une " grille de lecture » qu'il revendique, et surtout un terrain dans lequel il a passé bien plus de temps à enquêter et sur lequel il a davantage lu, un terrain pour lequel Berlin et Istanbul ont été des cas lui permettant de toujours mieux revenir à Paris (Fleury, 2008). Et c'est finalement le choix que nous avons fait nousmêmes de facto, Le Caire étant le terrain-pivot, et Oran le terrain servant de variable à la comparaison. Ainsi, nos enquêtes au Caire ont ainsi été plus longues, les terrains plus approfondis. Les séjours de recherche à Oran ont été plus ponctuels, plus concentrés, mais plus efficaces aussi. Finalement, partir à Oran a été bien souvent un moyen de mieux revenir au Caire, avec de nouvelles questions, de nouvelles idées. Faire ainsi une comparaison égale termes à termes entre deux terrains semble difficile à mener de front, en tout cas dans le temps imparti aujourd'hui à la réalisation d'une thèse en géographie. 

lisser les différences entre nos deux études de cas, en cherchant des invariants, les « traits communs » au-delà des spécificités des villes dont parle Philippe Gervais-Lambony (1994). Le géographe est conscient de ce risque et appelle à la prudence : le principal écueil de la comparaison est de "déformer les réalités pour faciliter des rapprochements ou des oppositions intellectuellement séduisantes [...]. Les réalités sont faciles à forcer et les conclusions doivent être prudentes» (ibid., 456). Marcel Detienne défend que «le comparatisme est plus vif qui prête l'oreille aux dissonances et que met en alerte l'incomparable. » (Detienne, 2002, 73), mais nous nous sommes rendu compte qu'une fois sur le terrain, il y avait une certaine facilité à remarquer les accords plutôt que les dissonances, pour filer la métaphore musicale. Par exemple, la question de la protection du patrimoine historique du centre-ville du Caire n'est apparue que tardivement, après un terrain égyptien et deux terrains algériens, alors qu'elle est centrale puisqu'elle détermine des règles d'urbanisme et donc de fabrique de la ville, au moins sur le papier. Or cette problématique est absente de notre terrain oranais, ce qui explique peut-être le temps pris pour prendre cette thématique en considération. Est-ce là le manque de prise en compte des différences, signifiantes par ailleurs, ou bien le travail de comparaison qui se met lentement en place? Comparer demande ainsi une flexibilité de l'esprit et de l'observation qu'il s'agit de retrouver en quittant un terrain pour l'autre.

\section{Conclusion : la construction de la comparaison par l'écriture}

Ainsi, mener une comparaison s'est révélé être au cours de ces trois années de recherche une méthodologie très riche qui nous aura permis de mener un travail de terrain dense, chaque fois renouvelé par les va-et-vient entre Oran et Le Caire. Mais il faut également noter que ces va-et-vient auront aussi été parfois des moments de malaise face aux difficultés de mise en œuvre de la comparaison.

La dernière difficulté du choix de cette méthode de travail réside désormais dans la mise par écrit de ce travail comparatif. En effet, les thèses comparatives en géographie urbaine que nous avons parcourues pour mettre au point notre méthodologie nous interpellent directement sur ce point : comment rédiger un travail comparatif ? Certaines d'entre elles annonçaient ainsi un travail de comparaison pour finalement proposer deux monographies posées face à face. D'autres, bien plus nombreuses heureusement, insistaient sur leur volonté de mettre en miroir les deux ou trois cas étudiés, afin de « dépasser la simple mise en parallèle [en imbriquant] à tous les niveaux d'analyse les deux espaces dans une analyse comparative » (Salin, 2002, 16), et passer d'un cas à l'autre. Néanmoins, cette juxtaposition des deux cas se fait finalement à l'intérieur des parties, car dans plusieurs thèses, les sous-parties ne concernent bien souvent qu'un seul cas sur les deux... Le problème n'est donc pas entièrement résolu !

L'écriture par thèmes, autrement dit par comparables, semble cependant la plus convaincante pour réaliser pleinement cette comparaison dans l'exercice final de la rédaction ainsi que pour permettre de monter en généralité. Comparer Le Caire et Oran doit ainsi permettre d'avancer sur la question des manifestations urbaines de phénomènes transnationaux que l'on trouve dans de multiples villes, et dans toutes les régions du monde, au Sud comme au Nord.

e-Migrinter, 13 | 2015 


\section{BIBLIOGRAPHIE}

Abaza, Mona (2006) Changing Consumer Cultures of Modern Egypt. Cairo's Urban Reshaping, Leiden; Boston, Brill, 309 p. (Social, Economic and Political Studies of the Middle East and Asia; vol. 101).

Bava, Sophie ; Pliez, Olivier (2009) Itinéraires d'élites musulmanes africaines au Caire. D’Al Azhar à l'économie de bazar, Afrique contemporaine, vol. 231, n³, pp. 187-207.

Belguidoum, Saïd (2011) Le dynamisme des nouvelles places marchandes de l'Est algérien : reconfiguration urbaine et nouvelles donnes sociales, Entre le licite et l'illicite : migration, travail, marchés, Cerisy, septembre 2011 [réf. du 5 mai 2015] [Disponible sur internet] (actes en cours de publication).

Belguidoum, Saïd ; Pliez, Olivier (2012) Construire une route de la soie entre l'Algérie et la Chine, Diasporas, $\mathrm{n}^{\circ} 20$, pp. 115-130.

Bellal, Samir (2008) Changement institutionnel et économie parallèle en Algérie : quelques enseignements, Revue du Chercheur, $\mathrm{n}^{\circ}$ 6, pp. 01-09.

Benbouzid, Mohamed (1999) Réseaux financiers et marchés parallèles de devises. Des Algériens dans l'économie informelle, Revue européenne de migrations internationales, vol. 15, $\mathrm{n}^{\circ} 2$, pp. 123-139.

Choplin, Armelle (2006) Fabriquer des villes-capitales entre monde arabe et Afrique noire: Nouakchott (Mauritanie) et Khartoum (Soudan), étude comparée, Paris, Université Paris 1 Panthéon-Sorbonne, $535 \mathrm{p}$.

Th. Doct : Géogr. : Paris : 2006.

Detienne, Marcel (2002) L'art de construire des comparables. Entre historiens et anthropologues, Critique internationale, $\mathrm{n}^{\circ} 14, \mathrm{pp} .68-78$.

Detienne, Marcel (2000) Comparer l'incomparable, Paris, Éd. du Seuil, 134 p.

Doron, Adrien (2006) Les réseaux transnationaux de commerce Sud/Sud. Exemple de trois nœuds d'échange en Égypte: Alexandrie, Le Caire et Nuweiba, Lyon, Université Lumière-Lyon 2, 136 p. Mém. M1 : Géogr : Lyon : 2006 (non publié).

Fleury, Antoine (2008) Croiser les terrains en géographie, A travers l'espace de la méthode : les dimensions du terrain en géographie, Arras, 18-20 juin 2008 [réf. du 11 juin 2014] [Disponible sur internet]

Fleury, Antoine (2007) Les espaces publics dans les politiques métropolitaines. Réflexions au croisement de trois expériences : de Paris aux quartiers centraux de Berlin et Istanbul, Paris, Université Paris 1 Panthéon Sorbonne, $685 \mathrm{p}$.

Th. Doct : Géogr. : Paris : 2007.

Gervais-Lambony, Philippe (2003) Territoires citadins. Quatre villes africaines, Paris, Belin, 271 p. (Mappemonde).

Gervais-Lambony, Philippe (1994) De Lomé à Harare. Le fait citadin. Images et pratiques de la ville, Paris, Karthala-IFRA, 472 p.

Igué, John O. ; Soulé, Bio G. (1992) L'Etat entrepôt au Bénin. Commerce informel ou solution à la crise? Paris, Karthala, 210 p. 
Kerdoud, Nadia (2012) Nouvelles centralités commerciales périphériques et recompositions territoriales. L'exemple des villes de l'Est algérien, Caen, Université de Caen, 390 p.

Th. Doct: Géogr.: Caen: 2012.

Mathews, Gordon; Alba Vega, Carlos (2012) Introduction, in Gordon, M.; et al. (dir.) Globalization From Below: the World's Other Economy, London; New York, Routledge, pp. 1-15.

Moisseron, Jean-Yves ; Youssef, Hoda (2004) Contraintes structurelles et ajustement par les changes en Égypte, Maghreb-Machrek, nº 182, pp. 13-36.

Morelle, Marie (2004) La rue des enfants, les enfants des rues, Paris, CNRS Éditions, 282 p.

Peraldi, Michel (2005) Routes algériennes, in Capron, G. ; Cortès, G. ; Guétat-Bernard, H. (dir.) Liens et lieux de la mobilité, Paris, Belin, pp. 273-290.

Peraldi, Michel (dir.) (2002) La fin des norias. Réseaux migrants dans les économies marchandes en Méditerranée, Paris, Maisonneuve et Larose, 495 p.

Peraldi, Michel (dir.) (2001) Cabas et containers. Activités marchandes informelles et réseaux migrants transfrontaliers, Paris, Maisonneuve et Larose, $361 \mathrm{p}$.

Pérouse, Jean-François (2007) Transits maghrébins à Istanbul : trajectoires, profils et stratégies, Revue des mondes musulmans et de la Méditerranée, n 119-120, pp. 115-138 [réf. du 14 avril 2011] [ Disponible sur internet]

Pliez, Olivier (2007) Des jeans chinois dans les rues du Caire, ou les espaces discrets de la mondialisation, Mappemonde vol. 88, n 4, [réf. du 11 juin 2014] [Disponible sur internet]

Salin, Elodie (2002) Les centres historiques du Caire et de Mexico : représentations de l'espace, mutations urbaines et protection du patrimoine, Nanterre, Université Paris X, 2 vol. (624 p.).

Th. Doct: Géogr.: Nanterre: 2002.

Simpfendorfer, Ben (2009) The New Silk Road: How a rising Arab world is turning away from the West and Rediscovering China, New York, Palgrave Macmillan, 208 p.

Spiga, Saskia (2002) Du nouveau système algérien d'importation aux nouvelles centralités commerciales dans la ville algérienne, in Peraldi, M. (dir.) La fin des norias ? Paris, Maisonneuve et Larose, pp. 217-242.

Spire, Amandine (2011) L'étranger et la ville en Afrique de l'Ouest. Lomé au regard d'Accra, Paris, Karthala, 378 p.

Tarrius, Alain (2002) La mondialisation par le bas. Les nouveaux nomades de l'économie souterraine, Paris, Balland, $168 \mathrm{p}$.

Tarrius, Alain (1995) Naissance d'une colonie : un comptoir commercial à Marseille, Revue européenne de migrations internationales, vol. 11, $\mathrm{n}^{\circ} 1$, pp. 21-52.

\section{NOTES}

1. En octobre 1986, la France impose en effet un régime de visa d'entrée aux ressortissants algériens, dans un contexte d'une vague d'attentats terroristes ayant eu lieu entre 1985 et 1986. Jusqu'à cette date, les ressortissants algériens pouvaient entrer en France sans visa. Cette fermeture économique et politique des frontières françaises, mais aussi européennes, a ainsi favorisé la recherche de nouvelles destinations commerciales (Belguidoum, 2011) et le développement d'autres places marchandes en Méditerranée (Peraldi, 2005). 
2. Pour des raisons évidentes de longueur de l'article, il ne sera développé dans cet article qu'un seul exemple de comparable afin de donner un aperçu rapide de la méthode utilisée pour la thèse.

3. Transcrit comme tel sur la devanture du magasin.

4. Entretien réalisé le 6 juin 2013 avec l'aide de Christina Samir, que nous remercions

5. Tandis que le taux de chômage de la population totale est estimé à 9,8 \% à la fin de l'année 2013 , il atteint chez les jeunes entre 16 et 24 ans un taux de $24,8 \%$, soit un jeune sur quatre. Source : ONS, Activités, emploi et chômage au $4^{\text {ème }}$ trimestre 2013, [réf. du 5 mai 2015], [Disponible sur internet].

6. À Alger ou à Constantine par exemple, le change se fait en pleine place publique. À Oran, il faut se rendre dans des boutiques en apparence banales (épiceries, boutiques de décoration, serrureries) mais dont l'arrière-boutique est entièrement dédiée au change parallèle.

INDEX

Mots-clés : méthodologie, espace urbain, petits commerces, circulation migratoire, mondialisation, étude comparative

Index géographique : Algérie, Oran, Égypte, Le Caire

\section{AUTEUR}

\section{ANNE BOUHALI}

Doctorante en géographie et aménagement, Université Toulouse 2 Jean-Jaurès, LISST-CIEU (UMR 5193) ; ATER à l'Université Paris Sorbonne anne.bouhali@univ-tlse2.fr 